\title{
KINETICS OF INTERLEUKIN-11 RELEASE AFTER CARDIOPULMONARY BYPASS
}

\author{
Daniel R. Meldrum, MD, David A. Partrick, MD, Brett C. Sheridan, MD, Ernest E. Moore, MD, and Alden H. Harken, MD,
} Denver, Colo

Cardiopulmonary bypass (CPB) is a proinflammatory state $^{1}$ that is associated with enhanced myocardial tumor necrosis factor- $\alpha$ (TNF) production. TNF is known to upregulate its own production, as well as the production of other inflammatory and anti-inflammatory cytokines. ${ }^{2}$ In this regard, interleukin-11 (IL-11) is a recently discovered multifunctional cytokine that belongs to the gp130 receptor subunit-linked family of anti-inflammatory cytokines. ${ }^{3}$ IL-11, a powerful stimulant of hematopoiesis, is clinically used to stimulate platelet production in patients with thrombocytopenia and is elevated in patients with disseminated intravascular coagulation. ${ }^{4}$ However, IL-11 also possesses potent antiinflammatory cytokine activity. Indeed, IL-11 decreases murine serum $\mathrm{TNF}^{5}$ and lung TNF production in response to endotoxin. ${ }^{6}$ Although our understanding of CPB-induced proinflammatory cytokine production is accelerating, ${ }^{1,2}$ little information exists regarding anti-inflammatory cytokine release, which may lead to late immunologic vulnerability. ${ }^{7}$ It remains unknown whether CPB induces the release of IL-11 and, if so, whether this correlates with the time course of late leukocyte disability. Enhanced understanding of the kinetics of IL-11 production after CPB may have important implications regarding the regulation of hematopoiesis, early inflammation, and late immunosuppression.

Methods. Patients, 18 to 65 years of age, scheduled for elective cardiac surgery but without other pre-existing disease were considered eligible for the study. The 6 patients enrolled underwent coronary artery bypass grafting at the University Hospital or Veterans Administration Medical Center on the University of Colorado Health Sciences Center Campus. Standard extracorporeal circulation was performed with a conventional membrane oxygenator, heat exchanger, and centrifugal pump with the patient under moderate hypothermia (systemic temperature between $28^{\circ} \mathrm{C}$ and $30^{\circ} \mathrm{C}$ ). Myocardial protection was afforded with cold blood cardioplegic solution $\left(4^{\circ} \mathrm{C}\right)$. The protocol to obtain serial blood samples was approved by the University of Colorado Health

From the Division of Cardiothoracic Surgery, Department of Surgery, University of Colorado, Denver, Colo.

Supported by National Institutes of Health grants HL-43696, HL44186, and GM-08315 and a National Institutes of Health NRSA.

Received for publication Jan 5, 1999; accepted for publication Feb 23, 1999.

Address for reprints: Daniel R. Meldrum, MD, Department of Surgery, 4200 East Ninth Ave, Box C-320, University of Colorado Health Sciences Center, Denver, CO 80262.

J Thorac Cardiovasc Surg 1999;118:193-5

Copyright (C) 1999 by Mosby, Inc.

$0022-5223 / 99 \$ 8.00+0 \quad \mathbf{1 2 / 5 4 / 9 8 0 7 6}$
Sciences Center Combined Affiliated Institutional Review Board, and written informed consent was obtained from all patients before enrollment. The first blood sample (baseline) was drawn during the patient's preoperative anesthesia appointment or, if the patient was in the hospital, the day before the operation. Samples were collected at the onset of CPB during the operation (time 0), and then 3, 6, 12, 24, and 36 hours after $\mathrm{CPB}$. Further samples were also obtained 2 and 3 days after CPB. At each time point, $15 \mathrm{~mL}$ of venous blood was collected into a heparinized syringe (10 units of heparin per milliliter of blood). The heparinized blood samples were centrifuged at $200 \mathrm{~g}$ for 15 minutes and the plasma was removed and stored at $-70^{\circ} \mathrm{C}$ until assay. A $50-\mathrm{mL}$ portion of each plasma sample was used in triplicate for the IL-11 assay, which was performed according to the manufacturer's instructions. In brief, the IL-11 assay uses the quantitative sandwich enzyme immunoassay technique. Standards and samples are loaded into the pre-coated wells (coated with monoclonal antibodies specific for IL-11) of a 96-well microtiter plate. Any IL-11 present in the plasma samples is bound by the monoclonal antibody bound to the well. After unbound sample is washed away, an enzyme-linked antibody specific for IL-11 is added to the wells. After another wash to remove unbound antibody, a color reagent is added that directly reflects the quantity of bound enzyme-linked anti-IL-11. The quantity is then read spectrophotometrically and is expressed as picograms per milliliter of a known standard. Standard reagents were obtained from Sigma Chemical Co, St Louis, Missouri, with the exception of the reagents for the IL-11 assay, which were obtained from R\&D Systems, Minneapolis, Minnesota. Data are reported as the mean \pm standard error of the mean for all groups tested. Analysis of variance was done with post hoc analysis using BonferroniDunn.

Results. The demographics, CPB time, number of coronary artery bypasses performed, and length of stay for the study patients are reported in Table I. A delayed sternal wound infection developed in 1 patient, necessitating a second hospital admission, wound debridement, and muscle flap closure. The remaining 5 patients had uneventful hospital courses and required no further hospitalizations related to their operation. The kinetics of IL-11 release after CPB are shown in Fig 1. IL-11 was undetectable in plasma obtained 0, 3,6 , and 12 hours after CPB. Twenty-four and 36 hours after CPB, IL-11 was detectable in plasma from some of the patients and undetectable in others, which yielded mean IL11 values of $2.8 \pm 1.8$ and $1.9 \pm 1.2 \mathrm{pg} / \mathrm{mL}$, respectively. At 48 hours, IL-11 was detectable in all patients, which resulted in a significant increase in mean IL-11 levels to $7.6 \pm 1.9$ 


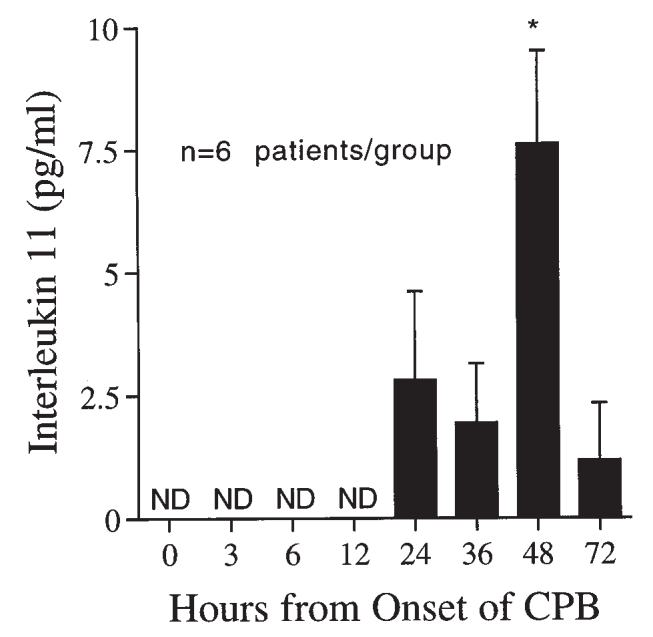

Fig 1. Kinetics of IL-11 release after CPB. Before CPB, IL11 was undetectable in the plasma of the 6 patients examined. After CPB, IL-11 was undetectable in plasma obtained at 3, 6, and 12 hours. However, 24 and 36 hours after CPB, IL-11 was detectable in plasma from some of the patients (2 of 6 patients at each time point; for 24 hours, $P=.07$; for 36 hours, $P=.2$ ). At 48 hours after CPB, IL-11 was detectable in all patients, which resulted in a significant increase in mean IL-11 levels (for 48 hours, $P=.0001$ ). At 72 hours after CPB, IL-11 levels declined (for 72 hours, $P=.05$ ).

pg/mL. Seventy-two hours after CPB, IL-11 levels had declined.

Discussion. The results of this study constitute the initial demonstration that CPB induces IL-11 production, which peaks at 48 hours. This peak in IL-11 production is temporally correlated with late post-CPB neutrophil disability. ${ }^{7}$ $\mathrm{CPB}$ is a routine part of cardiac surgery, and the vast majority of patients undergoing CPB recover uneventfully. In a small number of patients the "postperfusion syndrome" develops. This is characterized by diffuse inflammation, increased capillary permeability, extravasation of plasma, increased interstitial fluid, leukocytosis, fever, peripheral vasoconstriction, and a diffuse bleeding diathesis in its most severe form. Postperfusion syndrome is an exaggerated form of the systemic inflammatory response syndrome, recognized as an exuberant neutrophil/macrophage-mediated response. Recent studies have implicated myocardial TNF production and other proinflammatory cytokine production in the pathogenesis of this syndrome. ${ }^{1}$ Proinflammatory cytokines induce the later release of anti-inflammatory mediators, apparently intended to neutralize the inflammatory process in a negative feedback fashion. In its most severe form, excessive anti-inflammatory cytokine release can increase susceptibility to sepsis. ${ }^{1}$ IL-11 is a recently discovered pluripotent anti-inflammatory cytokine whose biologic impact is only beginning to be elucidated. IL-11 is a bone marrow fibroblast-derived cytokine. IL-11 has a variety of in
Table I. Clinical characteristics of patients undergoing $C P B(n=6$; means \pm standard deviation)

\begin{tabular}{lc}
\hline Mean age (y) & $50.0 \pm 5.7$ \\
Male & $6(100 \%)$ \\
CPB time (min) & $107 \pm 37.7$ \\
Crossclamp time (min) & $68 \pm 17.6$ \\
No. of CABGs & $3.0 \pm 1.1$ \\
Length of hospital stay (days) & $4.8 \pm 0.9$ \\
\hline
\end{tabular}

$C P B$, Cardiopulmonary bypass; $C A B G$, coronary artery bypass graft.

vitro biologic activities within the hematopoietic, lymphopoietic, hepatic, adipose, bone, gut, and neuronal systems. Because of the overlapping biologic activities of IL-11 and cytokines such as IL-6, leukemic inhibitory factor, ciliary neutrophilic factor, and oncostatin-M, it is not surprising to find that these cytokines share a common signal transducer, the gp130 common receptor subunit. Through the gp130 subunit, these cytokines disrupt the intracellular signaling of proinflammatory cytokines. Indeed, several investigators have demonstrated beneficial effects of IL-11 in experimental sepsis. ${ }^{6}$ Pretreatment with IL-11 significantly reduces mortality in a murine model of toxic shock syndrome, a rat model of experimental group B streptococcal sepsis, and a murine model of lethal endotoxemia. ${ }^{6}$ The antiinflammatory effects of IL-11 on both murine and rabbit models of endotoxemia appear to be due to the inhibition of the production of proinflammatory mediators. Trepicchio and coworkers ${ }^{5}$ demonstrated that IL-11 in a dose-dependent fashion reduced serum levels of pro-inflammatory mediators such as TNF, IL- $1-\beta$, and interferon- $\gamma$ after endotoxin administration in mice. These pretreatment strategies appear to inhibit injury-induced inflammation before its initiation. However, when released as a result of injury and proinflammatory cytokine release, IL-11 appears to be associated with late neutrophil disability. IL-11 production is temporally correlated with late post-CPB neutrophil disability. ${ }^{7}$ Given the role of IL-11 in platelet formation, early regulation of inflammation, and late immunosuppression, it appears as though regulating IL-11 production may be of future interest to cardiothoracic surgical investigation.

\section{REFERENCES}

1. Meldrum DR. Tumor necrosis factor in the heart (review). Am J Physiol 1998;274:R577-95.

2. Cameron D. Initiation of white cell activation during cardiopulmonary bypass: cytokines and receptors. J Cardiovasc Pharmacol 1996;27:S1-5.

3. Nandurkar HH, Hilton DJ, Nathan P, Willson T, Nicola N, Begley CG. The human IL-11 receptor requires gp130 for signaling: demonstration by molecular cloning of the receptor. Oncogene 1996;12:585-93.

4. Gordon MS, McCaskill-Stevens WJ, Battiato LA, et al. A phase I trial of recombinant human interleukin-11 (neumega rhIL-11 growth factor) in women with breast cancer receiving chemotherapy. Blood 1996;87:3615-24. 
5. Trepicchio WL, Bozza M, Dorner AJ. Recombinant human interleukin-11 attenuates the inflammatory response through downregulation of proinflammatory cytokine production and nitric oxide release. Blood 1995;86s:424a.

6. Sheridan BC, Dinarello CA, Meldrum DR, Selzman CH, Fullerton DA, McIntyre RC. Interleukin 11 attenuates cGMP mediated pulmonary vasomotor dysfunction during acute lung injury. Am J Physiol 1999. In press.

7. Partrick DA, Moore EE, Fullerton DA, Barnett CC, Meldrum DR, Silliman CC. Cardiopulmonary bypass renders patients at risk for multiple organ failure via early neutrophil priming and late neutrophil disability. J Surg Res 1999. In press. 